\title{
Vermehrung von Birnenbäumen der Sorte 'Uta' als Grünsteckling und Anbauverhalten im Vergleich zu Wurzelechten aus in vitro Vermehrung und auf zwei Unterlagen veredelten Bäumen unter biologischen Anbaubedingungen in Ostösterreich
}

\author{
Andreas Spornberger ${ }^{1} \cdot$ Gregor Osterc ${ }^{2}$ - Elisabeth Schüller ${ }^{1}$. Daniela Noll ${ }^{1}$
}

Eingegangen: 3. März 2019 / Angenommen: 12. März 2021 / Online publiziert: 20. April 2021

(c) Der/die Autor(en) 2021

\section{Zusammenfassung}

In den Jahren 2004 bis 2006 wurden jeweils im Sommer an der Universität Ljubljana Versuche zur Bewurzelung von Grünstecklingen der Birnensorte 'Uta' durchgeführt. Dabei bewurzelten zwischen 6,1 und 23,8\% der Langstecklinge. Im Herbst 2007 wurden die so erhaltenen wurzelechten Bäume im Versuchszentrum der Universität für Bodenkultur in Wien gepflanzt und mit wurzelechten Bäumen der Sorte 'Uta' aus in vitro-Vermehrung und mit auf Kirchensaller Mostbirne (Sämling) und Fox 11 veredelten verglichen. Alle Bäume wurden als Spindel erzogen und nach Bio-Anbau-Richtlinien gepflegt. Die wurzelechten Bäume kamen später in den Ertrag als die veredelten. Nach elf Jahren wiesen die Bäume auf Sämling den stärksten Wuchs und Ertrag auf, gefolgt von den Veredelungen auf Fox 11 und den wurzelechten Bäumen aus in vitro Vermehrung. Deutlich schwächer wuchsen die wurzelecht vermehrten Grünstecklinge. Keine Unterschiede gab es beim spezifischen Ertrag, der bei allen Varianten im Bereich zwischen 1,1 und 1,2 kg/ $\mathrm{cm}^{2} \mathrm{lag}$. Die nicht veredelten Bäume wiesen tendenziell größere Früchte als die beiden veredelten Varianten auf; speziell bei Fox 11 waren die Früchte kleiner. Wichtig bei den wurzelechten Bäumen ist die Verwendung kräftigerer Bäume bei der Pflanzung und die Durchführung einer Herbstpflanzung, um Baumausfälle in den ersten Jahren zu vermeiden.

Schlüsselwörter 'Uta' · Sämling · Fox 11 · Wurzelecht in vitro vermehrt · Wurzelechter Langsteckling

Andreas Spornberger

andreas.spornberger@boku.ac.at

1 Department of Crop Sciences, Institute of Viticulture and Pomology, University of Natural Resources and Life Sciences, Gregor Mendel Str. 33, 1180 Wien, Österreich

2 Department of Agronomy, Institute of Pomology, Viticulture and Vegetable Growing, University of Ljubljana, Ljubljana, Slowenien 


\title{
Propagation of Pear Trees of the cv. 'Uta' From Green Cuttings and Performance in the Field Compared to Own Rooted From in Vitro Propagation and Grafted Trees Under Organic Production Conditions in Eastern Austria
}

\begin{abstract}
From 2004 to 2006 propagation trials of green cuttings of the pear Cultivar 'Uta' were done at the University of Ljubljana. In the three-year summer trials between 6.1 and $23.8 \%$ of green cuttings were rooting. In autumn 2007 the obtained trees from the long cuttings were planted in the research center of the University of Natural Resources and Life Sciences in Vienna and compared with own rooted trees from in vitro propagation and with grafted trees on rootstock Kirchensaller Mostbirne (Seedling) and Fox 11. The training system was spindle, and the trees were treated according to organic production rules. The own rooted trees came later into bearing compared to the grafted ones. After eleven years, the trees on seedling showed the highest vigor and yield, followed by Fox 11 and the own rooted trees from in vitro propagation. The own rooted long cuttings grew clearly weaker. There were no differences in the specific yield, which was between 1.1 und $1.2 \mathrm{~kg}$ per $\mathrm{m}^{2}$. The own rooted trees showed larger fruits than the grafted ones. Especially Fox 11 had smaller fruits. For the own rooted ones it is important to use strong trees for plant material and to plant in autumn to avoid plant losses in the first years.
\end{abstract}

Keywords Propagation of long green cuttings - 'Uta' - Seedling · Fox $11 \cdot$ Own rooted trees from in vitro propagation . Own rooted trees from long cuttings

\section{Problemstellung}

Die Nachfrage nach biologisch produziertem Obst hat in den letzten Jahren einen stark positiven Trend erfahren. Die Bio-Anbaufläche in Österreich hat sich beim Apfel in den letzten fünf Jahren fast verdoppelt (2013: 792 ha, 2018: 1520 ha, AMA 2018). Im Vergleich dazu hat die Anbaufläche bei Birnen nur wenig zugenommen (2013: 141 ha, 2018: 177 ha, AMA 2018). Im biologischen Anbau in Österreich wurde in den letzten 15 Jahren vorwiegend die Birnensorte 'Uta', vor allem im Raum St. Pölten in Niederösterreich, ausgepflanzt (Rueß 2007). Was die Wahl geeigneter Unterlagen für den Bio-Anbau betrifft, gibt es aber, vor allem für Standorte mit hohem Kalkgehalt, wie in Niederösterreich und im Nordburgenland, noch viele offene Fragen.

Die im Intensivanbau aufgrund ihres schwachen Wuchses und frühen Ertrages verwendeten Quittenunterlagen $(\mathrm{C}$, A, Adams) sind für diese Standorte wenig geeignet, da es zu Eisenmangel und damit verbundener Chlorose kommt (Tomaz et al. 2009). Birnensämlinge, wie z. B. Kirchensaller Mostbirne, sind kalkverträglich, aber anfällig für Birnenverfall (Pear decline). Sie wachsen außerdem stark und beginnen spät zu fruchten (Tibiletti 2001). Fruchtgröße und Ausfärbung sind oft geringer als bei Quitten, die Neigung zu Wurzeltrieben, die anfällig für Feuerbrand (Erwinia amylovora) sind, dagegen ähnlich hoch. Die aus der Kreuzung der gegen Feuerbrand resistenten Sorte 'Old Home' mit 'Gute Luise" hervorgegangene Unterlage Pyrodwarf hat bei ähnlich starkem Wuchs wie der Sämling zum Teil kleinere Einzelfruchtgewichte gebracht und kann daher auch nicht empfohlen werden (Spornberger und Schüller 2016). Fox 11, eine neuere kalkverträgliche Birnenunterlage aus Italien, wird vom Wuchs als schwächer im Vergleich zum Sämling eingestuft, nur wenig stärker als die Unterlage BA 29 und soll für Pflanzungen zwischen 2000 und 2500 Bäumen je ha geeignet sein (Weber 2004), ist aber bei uns noch wenig untersucht worden.

In der Praxis des Bio-Birnenanbaus in Norditalien werden auch selbstbewurzelte Edelsorten aus in vitro Vermehrung erfolgreich verwendet. Sie sind von der Wuchsstärke dem Sämling ähnlich, ebenfalls spät generativ und gelten als robust gegen abiotische Schaderreger und Krankheiten, da sie auf eigener Wurzel stehen (Tibiletti 2001). Der spezifische Ertrag ist hoch (Thibault und Hermann 1982; Carrera und Gomez-Apparisi 2000; Stanica et al. 2000), die Fruchtgröße besser als beim Sämling (Thibault und Hermann 1982).

Über die Eignung von wurzelechten Birnenbäumen, die durch Bewurzelung von Langstecklingen auf eigener Wurzel gewonnen werden (Mbabu und Spethmann 2005; Spethmann 2007), gibt es, speziell im biologischen Anbau, keine Erfahrungen. Zusätzlich soll auch untersucht werden, inwieweit sich die Sorte 'Uta' grundsätzlich für diese Vermehrungsmethode eignet.

In einem vor kurzem abgeschlossenen Projekt wurden wurzelechte Birnenbäume aus in vitro Vermehrung bei den Sorten 'Bosc's', 'Williams Christ' und 'Uta' an mehreren Standorten in Österreich verglichen (Wurm et al. 2014; Spornberger und Schüller 2016; Steinbauer 2018). Dabei zeigten die selbstbewurzelten Bäume bei allen drei Sorten einen schwächeren Wuchs im Vergleich zu den Birnenunterlagen, und in Abhängigkeit von der Sorte einen späteren Ertragsbeginn. Bei 'Bosc's' war der Ertrag bei den Bäumen auf eigener Wurzel schwächer als bei den veredelten Vari- 
anten, bei 'Williams Christ' ähnlich. Bei 'Uta' waren die Erträge aufgrund einer ein Jahr später erfolgten Pflanzung nicht unmittelbar mit den veredelten vergleichbar.

Um nun für die im biologischen Anbau in Österreich wichtige Birnensorte 'Uta' entsprechende Daten zu bekommen, wurden in einem mehrjährigen Feldversuch selbst bewurzelte Bäume aus Grünstecklingen und in vitro Vermehrung mit auf Kirchensaller Sämling und Fox 11 veredelten Bäumen unter den Produktionsbedingungen des biologischen Anbaus hinsichtlich der Parameter Wuchs, Ertrag und Fruchtqualität verglichen. Außerdem wollten wir untersuchen, inwieweit sich 'Uta' für eine Bewurzelung von Langstecklingen eignet, wie es Spethmann (2007) bei diversen Obstarten propagiert.

\section{Material und Methoden}

\section{Gewinnung der Langstecklinge}

In den Jahren 2004 bis 2006 wurden Versuche zur Bewurzelung von Grünstecklingen durchgeführt. Die Triebe stammten von 'Uta'-Bäumen einer jungen Ertragsanlage der Baumschule Schreiber in Poysdorf und wurden sofort nach dem Schneiden in feuchte Tücher gewickelt und mit dem Auto an die Universität Ljubljana gebracht. Die Triebe wurden dort in einem Folienhaus unter Fog-System (Tropfengröße $10 \mu \mathrm{m})$ gesteckt. In Tab. 2 im Ergebnisteil ist der jeweilige genaue Zeitpunkt angeführt, wann die Triebe geschnitten und gesteckt wurden. Als Substrat wurde ein TorfSandgemisch im Verhältnis 3:1 mit einem $\mathrm{pH}-$ Wert von 4,0 verwendet, gedüngt mit 2,0 g/l Substrat Osmocote. Die Stecklinge wurden möglichst einheitlich auf ca. $60 \mathrm{~cm}$ Länge geschnitten. Direkt vor dem Stecken wurden die Schnittstellen in ein Gemisch auf Talkum-Basis mit 10\% Euparen und 0,5\% IBS (y-(3)-Indolyl-Buttersäure) getaucht. Ausgewertet wurde jeweils die Anzahl der bewurzelten Stecklinge im Spätherbst desselben Jahres.

Nach dem Verschulen der 2004 und 2005 gewonnen Stecklinge im jeweils darauf folgenden Frühling in die Baumschule im obstbaulichen Versuchszentrum der Universität für Bodenkultur in Wien musste ein Absterben der meisten Pflanzen festgestellt werden.

Um diese Pflanzausfälle zu vermeiden, wurden die im Sommer 2006 gewonnenen Pflanzen im Herbst 2007 zuerst einmal in Töpfe (Torf-Sandgemisch 3:1, gleiches Substrat wie bei der Vermehrung mit etwas Erde gemischt) gepflanzt und im ungeheizten Gewächshaus überwintert. Im Frühjahr 2007 wurden die Töpfe im Freien aufgestellt. Die auf diese Weise abgehärteten Pflanzen (Abb. 1) wurden dann im Herbst 2007 direkt ins Versuchsquartier ins Freiland für den nachfolgend beschriebenen Unterlagenversuch ausgepflanzt.

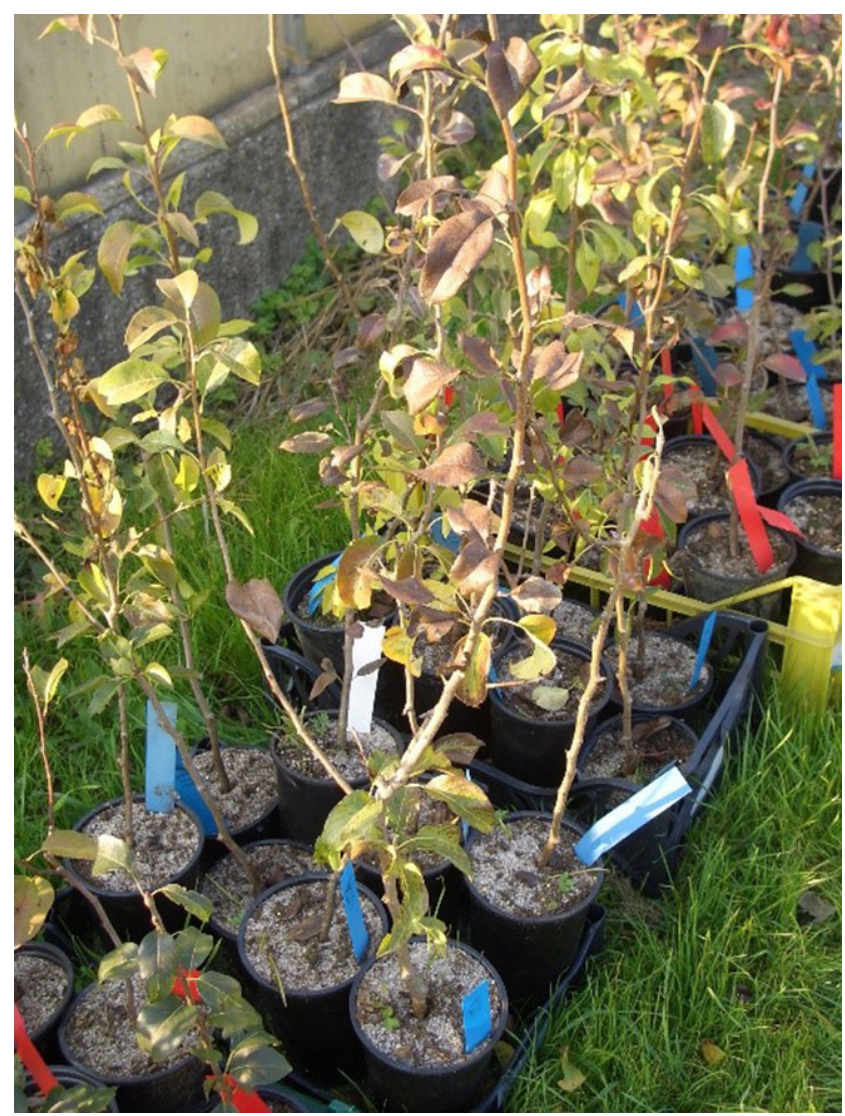

Abb. 1 Wurzelechte Langstecklinge der Sorte 'Uta' ein Jahr nach Aufschulung in Töpfen vor der Pflanzung ins Freiland im Herbst 2007. (Foto Spornberger)

\section{Unterlagenversuch}

Die Auspflanzung erfolgte im obstbaulichen Versuchszentrum der Universität für Bodenkultur in Jedlersdorf, am nordöstlichen Stadtrand von Wien. Die Jahresdurchschnittstemperatur am Standort beträgt ca. $10^{\circ} \mathrm{C}$, die durchschnittliche Niederschlagsmenge ca. $550 \mathrm{~mm}$. Der Boden ist ein tiefgründiger, leichter Tschernosem mit hohem Kalkgehalt (ca. 15\%), die Bodenreaktion leicht alkalisch (pH 7,5).

Als Versuchsbäume wurden im Herbst 2007 einjährige Okulanten der Sorte 'Uta' auf den Unterlagen Sämling Kirchensaller Mostbirne und Fox 11 von der Baumschule Schreiber (Poysdorf, Österreich) sowie einjährige und ein Jahr aufgeschulte, wurzelechte Bäume aus in vitro Vermehrung bzw. von den im Sommer 2006 bewurzelten Grünstecklingen (genauere Beschreibung weiter oben) verwendet (Tab. 1). Je Unterlage wurden 15 Bäume, aufgeteilt auf fünf Wiederholungsblöcke zu je drei Bäumen, verwendet. Die Baumerziehung erfolgte nach dem im Anbau üblichen Spindelsystem mit einem Pflanzabstand von $4 \times 2 \mathrm{~m}$.

Der Baumstreifen wurde mit einem Stockräumer mehrmals im Jahr mechanisch freigehalten. Pflanzenschutzmaßnahmen erfolgten nach den Richtlinien des biologischen 
Tab. 1 Varianten und Herkunft des Pflanzmaterials

\begin{tabular}{|c|c|c|}
\hline Variante & Herkunft & \\
\hline Fox 11 & Schreiber & 1j Okulant \\
\hline $\begin{array}{l}\text { Sämling Kir- } \\
\text { chensaller }\end{array}$ & Schreiber & 1j Okulant \\
\hline $\begin{array}{l}\text { Wurzelecht in } \\
\text { vitro }\end{array}$ & Italien über Schreiber & $\begin{array}{l}2 \mathrm{j}, 1 \mathrm{j} \text { in Baumschule } \\
\text { aufgeschult }\end{array}$ \\
\hline $\begin{array}{l}\text { Wurzelecht } \\
\text { Langsteckling }\end{array}$ & $\begin{array}{l}\text { Univ. Ljubljana, Triebe } \\
\text { von Schreiber }\end{array}$ & $\begin{array}{l}\text { 1j Steckling, 1j in } \\
\text { Topf aufgeschult }\end{array}$ \\
\hline
\end{tabular}

Anbaus, hauptsächlich wurden Netzschwefel und Granulosevirus sowie geringe Mengen Kupfer eingesetzt. Bewässert wurde in der Vegetationszeit nach Bedarf mit einer Überkronenberegnung. Sonstige Pflegemaßnahmen wie organische Düngung, Baumerziehung, Schnitt, und Mulchen der Fahrgasse wurden bei allen Varianten auf ähnliche Weise durchgeführt. Die Ernte erfolgte in einem Durchgang, wobei Gewicht und Fruchtzahl baumweise erfasst wurden. Im Winter wurde bei jedem Baum mit einem Maßband in $40 \mathrm{~cm}$ Höhe der Stammumfang gemessen. Der spezifische Ertrag wurde als kumulierter Einzelbaumertrag bezogen auf die errechnete Stammquerschnittsfläche im Herbst 2018 dargestellt.

In den Jahren 2010-2013 wurde in der zweiten Maihälfte eine Bonitur auf die tierischen Schädlinge Birnblattsauger (Psylla pirisuga Foerst, Psylla piri L.), Mehlige Birnenblattlaus (Dysaphis pyri Boyer de Fonscolombe) und Birnentriebwespe (Janus compressus F.) sowie Chlorose durchgeführt. Jeder Baum wurde dazu von zwei Personen von beiden Seiten auf Schaderreger und Symptome betrachtet und gemeinsam anhand einer Boniturskala von 0 (kein Befall/keine Chlorose) bis 5 (extrem starker Befall/extrem stark chlorotisch) bewertet. Die Symptome von Birnengitterrost (Gymnosporangium sabinae) wurden mit dem gleichen System im August bonitiert.

In den Jahren 2017 und 2018 wurden zur Ernte je 25 Früchte pro Variante von verschiedenen Bäumen, verteilt auf die Wiederholungen, vom Baum genommen und im Kühlraum bei $3{ }^{\circ} \mathrm{C}$ gelagert. Nach dem Auslagern wurden die Früchte einen Tag bei Zimmertemperatur nachreifen gelassen und dann im Labor untersucht auf: Fruchtgewicht (mit einer Laborwaage FA-2000S, Sartorius, Deutschland), Fruchtformindex errechnet aus Fruchtlänge ${ }^{2} /$ Fruchtbreite * Fruchtdicke, Fruchtfarbe mit dem L*a*b*-Messgerät (Konica Minolta, Japan), lösliche Trockensubstanz mit einem digitalen Refraktometer (Atago, Japan) in ${ }^{\circ}$ Brix bei $20^{\circ} \mathrm{C}$ (Khazaei et al. 2008). Die titrierbare Säure wurde mit dem Gerät TitroLinealpha plus (Schott, Deutschland) nach Wurm et al. (2005) ermittelt, wobei $10 \mathrm{ml}$ Probenvolumen mit $0,1 \mathrm{~mol} / 1 \mathrm{NaOH}$ bis zu pH 8,1 titriert wurden (Thybo et al. 2006). Daraus wurde der Säuregehalt errechnet: [g/l] Apfelsäure $=[\mathrm{ml}] \mathrm{NaOH} * 0,67$. Das Zucker-Säureverhältnis wurde mit folgender Formel errechnet: Brix * $10 /$ [g/l] Apfelsäure (OECD 2005).

Am 27.09.2018 wurde je eine Kiste mit ca. $25 \mathrm{~kg}$ pro Wiederholung sofort nach der Ernte abgewogen und im Kühlraum bei $2{ }^{\circ} \mathrm{C}$ eingelagert. Bei der Auslagerung am 12.02.2019 wurden Gewichtsverlust sowie \% gesunde und faule Früchte ermittelt.

Die statistische Auswertung der Daten erfolgte mit dem Statistikprogramm SPSS (Version 24, IBM, Österreich). Nach Prüfung der Daten auf Varianzhomogenität und Normalverteilung wurde eine Varianzanalyse in Verbindung mit einem F-Test durchgeführt, um die Mittelwerte anschließend mittels Grenzdifferenz nach S-N-K zu beurteilen, wobei generell mit einem Signifikanzniveau $p<0,05$ gearbeitet wurde.

\section{Ergebnisse}

\section{Gewinnung der Langstecklinge}

In Tab. 2 ist der jeweilige genaue Zeitpunkt angeführt, wann die Triebe geschnitten und gesteckt wurden und wieviel Prozent der Triebe im Herbst desselben Jahres bewurzelt waren. Die Bewurzelung schwankte zwischen 6,1\% im Jahr 2004 und 23,8\% im Jahr 2006.

\section{Baumausfälle}

Im Pflanzjahr fielen je zwei Bäume der Varianten Fox 11 und wurzelechter Langsteckling aus sowie einer der wurzelecht in vitro vermehrten. Diese Bäume wurden im Herbst 2008 mit gleichwertigen Bäumen ersetzt. Bis zum Ende des Versuches im Jahr 2018 waren wiederum je zwei Bäume bei Fox 11 und den wurzelechten Langstecklingen sowie ein Baum bei den wurzelecht in vitro vermehrten Bäumen abgestorben. Zusätzlich wiesen je ein Baum auf Fox 11 und auf Kirchensaller Sämling in den letzten beiden Jahren die für Birnenverfall (Pear decline) typischen Krankheitssymptome (extrem kleine Früchte) auf (Tab. 3).
Tab. 2 Anzahl Steckhölzer und Prozent Bewurzelung von Grünstecklingen der Sorte 'Uta' an der Universität Ljubljana in den Jahren 2004-2006

\begin{tabular}{lllll}
\hline Jahr & $\begin{array}{l}\text { Steckhölzer } \\
\text { Anzahl }\end{array}$ & Geschnitten am & Gesteckt am & $\begin{array}{l}\text { Bewurzelung im Herbst } \\
\%\end{array}$ \\
\hline 2004 & 500 & 14.07. morgens & 14.07. nachmittag & 6,1 \\
2005 & 240 & 29.06. morgens & 29.06. abends & 10,4 \\
2006 & 240 & 18.07. abends & 19.07. vormittag & 23,8 \\
\hline
\end{tabular}


Tab. 3 Baumausfälle der Sorte 'Uta' veredelt auf den Unterlagen Fox 11 und Kirchensaller Sämling, sowie wurzelecht in vitro und als Langsteckling bis Ende der Jahre 2008 und 2018

\begin{tabular}{llll}
\hline & \multicolumn{2}{c}{$\%$ Baumausfälle } & $\begin{array}{l}\text { \% Krank } \\
\text { (Pear decline) }\end{array}$ \\
\cline { 2 - 3 } & $\begin{array}{l}\text { Bis } \\
\text { Bis 2018 }\end{array}$ & Bis & Bis \\
& 2008 & 2018 & \\
\hline Fox 11 & 13,3 & 13,3 & 6,7 \\
Sämling Kirchensaller & 0,0 & 0,0 & 6,7 \\
Wurzelecht in vitro & 6,7 & 6,7 & 0,0 \\
Wurzelecht Langsteckling & 13,3 & 13,3 & 0,0 \\
\hline
\end{tabular}

\section{Chlorose, Krankheiten und Schädinge}

Bei den tierischen Schädlingen Birnblattsauger und Mehlige Birnenblattlaus sowie bei der Chlorose konnten keine wesentlichen Unterschiede zwischen den Kombinationen festgestellt werden. Bei der Birnentriebwespe (Janus compressus) war im Jahr 2013 deutlich weniger Befall bei den wurzelechten Langstecklingen im Vergleich zu den anderen Varianten zu beobachten. Auch beim Birnengitterrost (Gymnosporangium sabinae Winter) zeigten diese Bäume in drei von vier Jahren einen geringeren Befall als die anderen Varianten (Tab. 4).

\section{Wuchs und Ertrag}

Den stärksten Wuchs zeigten die auf Sämling veredelten Bäume. Am zweitstärksten waren die Veredelungen auf Fox 11, und zwar gleich auf mit den wurzelechten Bäumen aus in vitro Vermehrung. Deutlich schwächer wuchsen die wurzelecht vermehrten Langstecklinge (Tab. 5).

Die wurzelechten Bäume kamen, verglichen mit den Veredelungen auf Fox 11 und Sämling, später in den Ertrag (Abb. 2). Die Bäume auf Sämling wiesen mit knapp $84 \mathrm{~kg}$ den höchsten Einzel-Baumertrag auf, gefolgt von Fox 11 $(60,8 \mathrm{~kg})$, wurzelecht in vitro $(55,1 \mathrm{~kg})$ und wurzelechter Langsteckling $(40,5 \mathrm{~kg})$. Letztere drei unterschieden sich statistisch nicht signifikant voneinander (Tab. 5).

Wird die Wuchsstärke miteinbezogen, so ergeben sich bei Betrachtung der spezifischen Fruchtbarkeit keine signifikanten Unterschiede zwischen den Varianten. Der spezifische Ertrag liegt bei allen Varianten im Bereich zwischen 1,1 und $1,2 \mathrm{~kg} / \mathrm{cm}^{2}$ (Tab. 3).

Tab. 4 Ergebnisse der Bonituren auf Eisenchlorose, Birnengitterrost und diverse Schaderreger in den Jahren 2010-2013

\begin{tabular}{|c|c|c|c|c|c|c|c|c|}
\hline & 2010 & $*$ & 2011 & $*$ & 2012 & $*$ & 2013 & $*$ \\
\hline \multicolumn{9}{|l|}{ Chlorose } \\
\hline Fox 11 & 0,00 & a & 0,33 & a & 0,27 & a & n.b. & - \\
\hline Sämling Kirchensaller & 0,00 & a & 0,07 & a & 0,07 & a & n.b. & - \\
\hline Wurzelecht in vitro & 0,07 & a & 0,20 & a & 0,00 & a & n.b. & - \\
\hline Wurzelecht Langsteckling & 0,00 & a & 0,31 & a & 0,14 & a & n.b. & - \\
\hline \multicolumn{9}{|l|}{ Birnengitterrost (G. sabinae) } \\
\hline Fox 11 & 0,50 & $\mathrm{~b}$ & 1,00 & $\mathrm{~b}$ & 1,07 & a & 0,93 & $\mathrm{~b}$ \\
\hline Sämling Kirchensaller & 0,50 & $\mathrm{~b}$ & 1,07 & $\mathrm{~b}$ & 1,07 & a & 1,00 & $\mathrm{~b}$ \\
\hline Wurzelecht in vitro & 0,46 & $\mathrm{~b}$ & 0,93 & $\mathrm{~b}$ & 1,13 & a & 0,87 & $a b$ \\
\hline Wurzelecht Langsteckling & 0,36 & a & 0,69 & a & 1,07 & a & 0,64 & a \\
\hline \multicolumn{9}{|l|}{ Birnblattsauger (Psylla ssp.) } \\
\hline Fox 11 & 0,67 & $\mathrm{a}$ & 0,47 & a & 1,53 & a & 0,73 & a \\
\hline Sämling Kirchensaller & 0,73 & a & 1,10 & a & 2,00 & a & 1,13 & a \\
\hline Wurzelecht in vitro & 0,50 & a & 0,60 & a & 1,80 & a & 0,93 & a \\
\hline Wurzelecht Langsteckling & 0,50 & a & 1,00 & a & 1,64 & a & 1,36 & a \\
\hline \multicolumn{9}{|c|}{ Mehlige Birnblattlaus (Dysaphis pyri) } \\
\hline Fox 11 & 0,07 & $\mathrm{a}$ & 0,07 & a & 0,27 & a & 0,33 & a \\
\hline Sämling Kirchensaller & 0,00 & a & 0,00 & a & 0,33 & a & 1,07 & a \\
\hline Wurzelecht in vitro & 0,00 & a & 0,00 & a & 0,20 & a & 0,53 & a \\
\hline Wurzelecht Langsteckling & 0,00 & a & 0,00 & a & 0,07 & $\mathrm{a}$ & 0,71 & a \\
\hline \multicolumn{9}{|c|}{ Birnentriebwespe (J. compressus) } \\
\hline Fox 11 & n.b. & - & 0,47 & a & 1,33 & a & 1,13 & $\mathrm{~b}$ \\
\hline Sämling Kirchensaller & n.b. & - & 0,83 & a & 1,47 & a & 1,47 & $\mathrm{~b}$ \\
\hline Wurzelecht in vitro & n.b. & - & 0,67 & a & 1,67 & a & 1,14 & $\mathrm{~b}$ \\
\hline Wurzelecht Langsteckling & n.b. & - & 1,00 & $\mathrm{a}$ & 1,64 & $\mathrm{a}$ & 0,57 & a \\
\hline
\end{tabular}

Boniturskala von $0=$ kein Befall/keine Chlorose bis $5=$ extrem starker Befall/extrem stark chlorotisch $n . b$. nicht bonitiert

*Verschiedene Buchstaben weisen auf signifikante Unterschiede hin (ANOVA mit nachfolgendem S-N-K-Test bei $p<0,05$ ) 
Tab. 5 Summe der Erträge 2008-13, Stammquerschnitt und spezifischer Ertrag der Sorten Bosc's, Williams und 'Uta' auf den verschiedenen Unterlagen
Abb. 2 Mittlerer jährlicher Baumertrag von 'Uta' auf den verschiedenen Unterlagen in den Jahren 2009 bis 2018

\begin{tabular}{|c|c|c|c|c|c|c|}
\hline & \multicolumn{2}{|c|}{$\begin{array}{l}\text { Stammquerschnitt } \\
\text { Nov. } 2018\end{array}$} & \multicolumn{2}{|c|}{$\begin{array}{l}\text { Summe Erträge } \\
2009-18\end{array}$} & \multicolumn{2}{|c|}{ Spez. Ertrag summiert } \\
\hline & $\overline{\mathrm{cm}^{2}}$ & $*$ & $\mathrm{~kg} / \mathrm{Baum}$ & $*$ & $\mathrm{~kg} / \mathrm{cm}^{2}$ & $*$ \\
\hline Fox 11 & 54,6 & $\mathrm{~b}$ & 60,8 & $\mathrm{a}$ & 1,14 & $\mathrm{a}$ \\
\hline Sämling Kirchensaller & 68,0 & $\mathrm{c}$ & 83,8 & $\mathrm{~b}$ & 1,22 & $\mathrm{a}$ \\
\hline Wurzelecht in vitro & 53,3 & $\mathrm{~b}$ & 55,1 & $\mathrm{a}$ & 1,12 & a \\
\hline Wurzelecht Langsteckling & 31,8 & a & 40,5 & a & 1,23 & a \\
\hline
\end{tabular}

*Verschiedene Buchstaben weisen auf signifikante Unterschiede hin (ANOVA mit nachfolgendem S-N-KTest bei $p<0,05$ )

$\mathrm{kg} /$ Baum

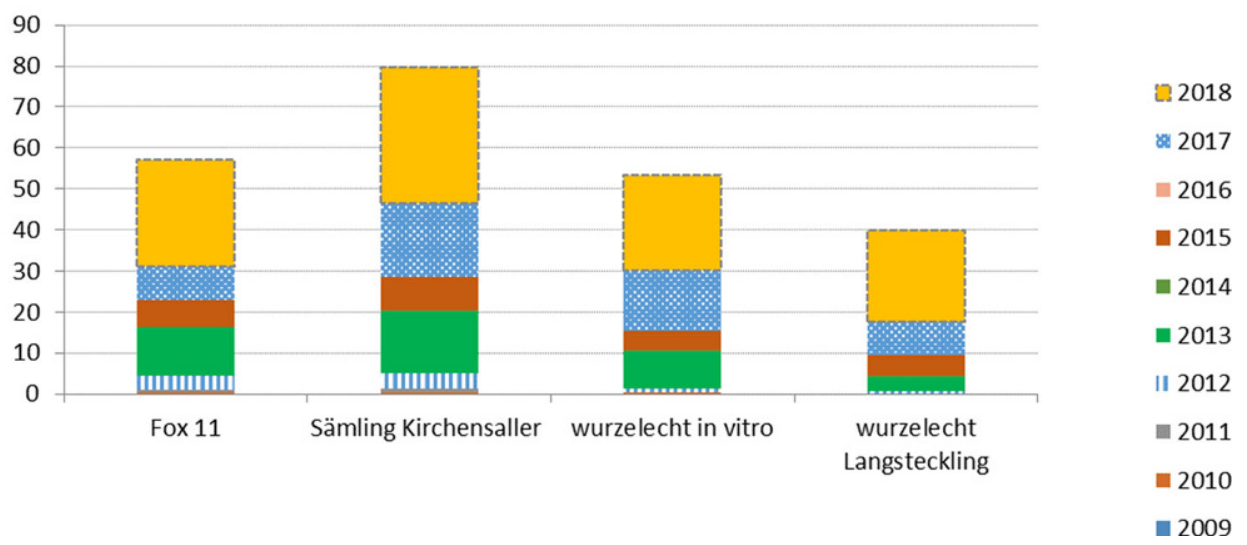

Tab. 6 Mittleres Fruchtgewicht (g/Stück) von 'Uta' bei der Ernte (Mittelwerte der Jahre 2015, 2017 und 2018) auf den Unterlagen Fox 11, Kirchensaller Sämling, sowie wurzelecht in vitro und als Grünsteckling

\begin{tabular}{lllllll}
\hline & \multicolumn{7}{l}{ Mittleres Stückgewicht in g/Frucht } \\
\cline { 2 - 7 } & 2015 & & 2017 & & 2018 & \\
\hline Fox 11 & 255 & $\mathrm{a}$ & 133 & $\mathrm{a}$ & 168 & $\mathrm{a}$ \\
$\begin{array}{l}\text { Sämling Kir- } \\
\text { chensaller }\end{array}$ & 235 & $\mathrm{a}$ & 148 & $\mathrm{ab}$ & 210 & $\mathrm{~b}$ \\
$\begin{array}{l}\text { Wurzelecht in } \\
\text { vitro }\end{array}$ & 290 & $\mathrm{a}$ & 170 & $\mathrm{~b}$ & 198 & $\mathrm{~b}$ \\
$\begin{array}{l}\text { Wurzelecht } \\
\text { Langsteckling }\end{array}$ & 281 & $\mathrm{a}$ & 167 & $\mathrm{~b}$ & 229 & $\mathrm{~b}$ \\
\hline
\end{tabular}

*Verschiedene Buchstaben weisen auf signifikante Unterschiede hin (ANOVA mit nachfolgendem S-N-K-Test bei $p<0,05$ )

\section{Fruchtqualität}

Die Früchte auf Fox 11 waren in den Jahren 2017 und 2018 deutlich kleiner und leichter als die der beiden wurzelechten Varianten. Im Jahr 2018 waren sie auch leichter als die Früchte auf Sämling. Zwischen den anderen Varianten gab es keine signifikanten Unterschiede, obwohl die Wurzelechten in zwei Jahren (2015 und 2017) tendenziell größere Früchte aufwiesen wie die Veredelungen auf Sämling (Tab. 6).

Bei den Untersuchungen im Labor waren im ersten Jahr die untersuchten Früchte bei den in vitro Bäumen signifikant größer als auf Fox 11 und Sämling. Bei den Farbmes- sungen im Jahr 2017 wiesen die wurzelechten Langstecklinge signifikant grünere Früchte auf (a*-Anteil) im Vergleich $\mathrm{zu}$ allen anderen Varianten und einen höheren Blau-Anteil (b*) im Vergleich zur Variante Fox 11 auf. Diese Farb-Differenzen konnten im Jahr 2018 nicht bestätigt werden. In allen anderen Messparametern gab es keine Unterschiede zwischen den Varianten (Tab. 7).

\section{Lagerung}

Die Gewichtsverluste nach ca. 4,5 Monaten Kühllagerung im Jahr 2018 bewegten sich zwischen 7,0 und 7,8\%. Der Anteil verkaufsfähiger Früchte lag zwischen $75,0 \%$ bei den wurzelechten Langstecklingen und 78,5\% bei den wurzelechten aus in vitro Vermehrung. Die beobachteten Unterschiede waren allesamt nicht gesichert (Tab. 8).

\section{Diskussion}

Die Vermehrung von Birnenedelsorten mittels Grünstecklingen im Sommer ist machbar. Die Bewurzelung lag zwischen 6,1 und 23,8\%, also geringer als bei Mbabu und Spethmann (2005), die bei 'Williams Christ' Bewurzelungserfolge zwischen 30 und $50 \%$ erzielten. Bei der direkten Verschulung der bewurzelten Stecklinge ins Freiland im nachfolgenden Frühjahr waren zum Teil sehr starke Pflanzausfälle festzustellen. Es zeigte sich jedoch, dass dieses 
Tab. 7 Ergebnisse der 2017 und 2018 durchgeführten Fruchtqualitätsmessungen im Labor (Mittelwerte in den beiden Jahren)

\begin{tabular}{|c|c|c|c|c|c|c|c|c|c|c|c|c|c|c|c|c|}
\hline & \multicolumn{2}{|c|}{ Fruchtgewicht } & \multicolumn{6}{|c|}{ Fruchtfarbe } & \multicolumn{2}{|c|}{ Fruchtformindex } & \multicolumn{2}{|c|}{$\begin{array}{l}\text { Lösliche Tro- } \\
\text { ckensubstanz }\end{array}$} & \multicolumn{2}{|c|}{ Apfelsäure } & \multicolumn{2}{|c|}{$\begin{array}{l}\text { Zucker/ } \\
\text { Säure }\end{array}$} \\
\hline & $\begin{array}{l}\text { g/ } \\
\text { Frucht }\end{array}$ & $*$ & $\mathrm{~L}^{*}$ & $*$ & $a^{*}$ & $*$ & $b^{*}$ & $*$ & & $*$ & $\begin{array}{l}\% \\
\text { Brix }\end{array}$ & $*$ & $\mathrm{~g} / \mathrm{l}$ & $*$ & & $*$ \\
\hline \multicolumn{17}{|l|}{2017} \\
\hline Fox 11 & 175 & a & 60,8 & $\mathrm{a}$ & 13,3 & $\mathrm{~b}$ & 45,2 & $\mathrm{~b}$ & 1,16 & a & 18,3 & a & 3,29 & a & 57,0 & $\mathrm{a}$ \\
\hline $\begin{array}{l}\text { Sämling } \\
\text { Kirchensal- } \\
\text { ler }\end{array}$ & 170 & $\mathrm{a}$ & 51,7 & $\mathrm{a}$ & 10,2 & $\mathrm{~b}$ & 33,3 & $a b$ & 1,15 & a & 17,0 & $\mathrm{a}$ & 3,11 & a & 53,8 & $\mathrm{a}$ \\
\hline $\begin{array}{l}\text { Wurzelecht } \\
\text { in vitro }\end{array}$ & 231 & $\mathrm{~b}$ & 58,6 & $\mathrm{a}$ & 10,2 & $\mathrm{~b}$ & 40,3 & $a b$ & 1,10 & a & 16,4 & a & 3,22 & a & 53,1 & $\mathrm{a}$ \\
\hline $\begin{array}{l}\text { Wurzelecht } \\
\text { Langsteck- } \\
\text { ling }\end{array}$ & 210 & $a b$ & 41,7 & a & $-13,0$ & $\mathrm{a}$ & 17,8 & $\mathrm{a}$ & 1,35 & a & 17,9 & a & 2,86 & a & 63,1 & $\mathrm{a}$ \\
\hline 2018 & & & & & & & & & & & & & & & & \\
\hline Fox 11 & 156 & a & 50,8 & $\mathrm{a}$ & 10,5 & a & 33,5 & $\mathrm{a}$ & 0,85 & a & 16,4 & $\mathrm{a}$ & 1,84 & $\mathrm{a}$ & 88,6 & $\mathrm{a}$ \\
\hline $\begin{array}{l}\text { Sämling } \\
\text { Kirchensal- } \\
\text { ler }\end{array}$ & 254 & $\mathrm{a}$ & 50,7 & $\mathrm{a}$ & 10,9 & $\mathrm{a}$ & 34,1 & $\mathrm{a}$ & 1,01 & $\mathrm{a}$ & 15,1 & $\mathrm{a}$ & 2,35 & $\mathrm{a}$ & 67,0 & $\mathrm{a}$ \\
\hline $\begin{array}{l}\text { Wurzelecht } \\
\text { in vitro }\end{array}$ & 193 & a & 49,6 & $\mathrm{a}$ & 8,1 & a & 32,6 & $\mathrm{a}$ & 1,09 & a & 14,8 & a & 2,65 & a & 62,2 & $\mathrm{a}$ \\
\hline $\begin{array}{l}\text { Wurzelecht } \\
\text { Langsteck- } \\
\text { ling }\end{array}$ & 181 & $\mathrm{a}$ & 52,9 & $\mathrm{a}$ & 9,3 & a & 38,9 & a & 1,04 & $\mathrm{a}$ & 15,6 & $\mathrm{a}$ & 2,13 & a & 74,9 & $\mathrm{a}$ \\
\hline
\end{tabular}

*Verschiedene Buchstaben weisen auf signifikante Unterschiede hin (ANOVA mit nachfolgendem S-N-K-Test bei $p<0,05$ )

Tab. 8 Ergebnisse des Lagerversuches der Ernte 2018 bei der Auslagerung am 12.02.2019

\begin{tabular}{|c|c|c|c|c|c|c|}
\hline & \multicolumn{2}{|c|}{ Gewichtsverlust } & \multicolumn{2}{|c|}{ Vermarktbare Früchte } & \multicolumn{2}{|l|}{ Nicht vermarktbar } \\
\hline & $\%$ & $*$ & $\%$ Gewicht & $*$ & $\%$ Früchte (Anzahl) & $*$ \\
\hline Fox 11 & 7,7 & a & 75,9 & $\mathrm{a}$ & 18,3 & $\mathrm{a}$ \\
\hline $\begin{array}{l}\text { Sämling Kirchensal- } \\
\text { ler }\end{array}$ & 7,0 & $\mathrm{a}$ & 78,2 & a & 15,4 & $\mathrm{a}$ \\
\hline Wurzelecht in vitro & 7,8 & $\mathrm{a}$ & 78,5 & a & 13,7 & $\mathrm{a}$ \\
\hline $\begin{array}{l}\text { Wurzelecht Lang- } \\
\text { steckling }\end{array}$ & 7,4 & $\mathrm{a}$ & 75,0 & a & 19,0 & a \\
\hline
\end{tabular}

*Verschiedene Buchstaben weisen auf signifikante Unterschiede hin (ANOVA mit nachfolgendem S-N-KTest bei $p<0,05)$
Problem durch Überwinterung in einem ungeheizten Glashaus (Mbabu und Spethmann 2005; Spethmann 2007), bzw. nachfolgende einjährige Aufschulung in Töpfen beseitigt werden kann. Insgesamt ist das System verbesserungsfähig, um diese Form der Vermehrung wirtschaftlich zu machen. Hierzu sind weitere Untersuchungen nötig, um abzuklären, wann bei verschiedenen Obstarten der geeignete Zeitpunkt zur Vermehrung vorliegt und wie weit die zum Stecken verwendeten Triebe bereits verholzt sein dürfen.

Die in unserem Versuch durchgeführte Herbstpflanzung führte im ersten Jahr insgesamt zu deutlich weniger Ausfällen, verglichen mit einem ähnlichen Projekt am selben Standort, wo im Frühjahr gepflanzt worden war, und im Pflanzjahr speziell bei den in vitro Bäumen auf eigener Wurzel, die sehr wenig Feinwurzeln aufwiesen, hohe Baumverluste festgestellt wurden (Spornberger und
Schüller 2016). Die in unserem Versuch verwendeten Langstecklinge waren relativ schwach bei der Pflanzung. Zwei Bäume fielen gleich im Pflanzjahr aus, zwei weitere später. Bei dieser Variante ist daher eine längere Aufschulung in der Baumschule zu empfehlen, um stärkere Bäume zu erzielen. Gleich hoch war die Ausfallsquote auch bei der neuen Birnenunterlage Fox 11, jeweils 13\% sowohl im Pflanzjahr als auch nach 10 Jahren. Je ein Baum im Pflanzjahr und einer in den folgenden Jahren fiel bei den selbstbewurzelten Bäumen aus in vitro Vermehrung aus. Keine Ausfälle gab es nur bei Kirchensaller Sämling. Zu Versuchsende zeigten allerdings auch ein Baum dieser Unterlage und einer von Fox 11 Fruchtsymptome von Birnenverfall.

Bei der Bonitur von Schaderregern gab es wenig Unterschiede zwischen den Unterlagen. Die am schwächsten wachsende Variante Langsteckling zeigte in drei Jahren we- 
niger Symptome von Birnengitterrost im Vergleich zu allen anderen Varianten, und in einem Jahr weniger Birnentriebwespe. Ähnliche Beobachtungen beim Befall mit tierischen Schädlingen waren in dem früheren Projekt bei schwächer wachsenden Quittenunterlagen festgestellt worden (Spornberger und Schüller 2016), was wiederum den Schluss bestätigt, dass stärker wüchsige Bäume von einigen Schadinsekten und auch -pilzen stärker befallen werden als schwach wüchsige.

Den stärksten Wuchs und Einzelbaumertrag zeigten die Sämlinge, gefolgt von Fox 11 und den wurzelechten Bäumen aus in vitro Vermehrung, die etwa gleich stark wuchsen. Deutlich schwächer waren die wurzelecht vermehrten Langstecklinge. Der spezifische Ertrag ergab hingegen keine Unterschiede zwischen den Varianten.

Bei den Messungen der Fruchtqualität war ein geringeres Fruchtgewicht bei der Unterlage Fox 11 in zwei von drei Jahren zu beobachten. Die Unterschiede waren jedoch weniger stark ausgeprägt als bei Quitte Adams in einem früheren Projekt auf dem gleichen Standort (Spornberger und Schüller 2016). Die in einem Jahr bei den Früchten der Langstecklinge gemessenen Unterschiede bei der Fruchtfarbe konnten im zweiten Untersuchungsjahr nicht bestätigt werden.

Die Unterlage Kirchensaller Mostbirne zeigte den stärksten Wuchs und, wie schon in den vorhergehenden Arbeiten, mit 'Uta' den höchsten Einzelbaumertrag von allen Varianten (Wurm et al. 2014; Spornberger und Schüller 2016; Steinbauer 2018). Die im Rahmen des Bio-Uta-Projekts seit 2004 vor allem auf den Praxisbetrieben in Niederösterreich auf kalkreichen Böden gepflanzten Bäume, stehen fast ausschließlich auf Sämling, was aufgrund dieser Ergebnisse eine gute Unterlagenwahl war. Die in der Praxis und auch bei uns beobachtete Anfälligkeit für Birnenverfall - wir konnten einen kranken Baum nach zehn Jahren finden - muss bei dieser Unterlage allerdings im Auge behalten werden.

Fox 11 brachte im Vergleich zum Sämling eine signifikante Wuchsreduktion, die sich allerdings auch in einem geringeren Einzelbaumertrag - bei gleichem spezifischem Ertrag - niederschlug. Bei dieser Unterlage wurde zumindest in einem Jahr ein geringeres Stückgewicht im Vergleich zum Sämling festgestellt. Sowohl im Pflanzjahr als auch nach zehn Jahren gab es Baumausfälle. Aufgrund dieser Ergebnisse kann die Unterlage nicht als schwächer wüchsiger Ersatz zum Sämling - zumindest für die Sorte 'Uta' empfohlen werden.

Die wurzelechten Bäume aus in vitro Vermehrung wuchsen schwächer als die Sämlinge, etwa gleich wie Fox 11. Allerdings kamen sie verglichen mit den veredelten Bäumen später in den Ertrag, Dies entspricht den Ergebnissen mit Birnenbäumen auf eigener Wurzel aus in vitro Vermehrung im Vergleich zu veredelten Bäumen aus ähnlichen Versuchen (Wurm et al. 2014; Spornberger und Schüller 2016) und hängt vermutlich mit dem juvenilen Charakter dieser Bäume zusammen. Die aus Grünstecklingen gewonnenen selbstbewurzelten Bäume waren bei der Pflanzung schwach und wuchsen auch in den weiteren Jahren deutlich schwächer im Vergleich zu den anderen Varianten. Dennoch kamen sie um ein bis zwei Jahre später in die generative Phase als die veredelten Bäume. Diese Ergebnisse stehen im Gegensatz zu jenen von Mbabu und Spethmann (2005), die berichteten, dass Birnenstecklinge von 'Williams Christ' sehr schnell nach dem Vermehrungsjahr in den Ertrag kamen und schon im ersten Verschulungsjahr Blüten zeigten.

Bei beiden wurzelechten Varianten war eine zum Teil bessere Fruchtgröße als bei den veredelten als positiv zu vermerken. Außerdem gab es im Gegensatz zu den veredelten Bäumen keine durch Birnenverfall befallenen Bäume.

Besonders bei den Langstecklingen ist eine längere Aufschulung in der Baumschule zu empfehlen, um kräftigere Bäume zu erzielen. Bei den in vitro Bäumen hat sich in einem anderen Versuch gezeigt (Spornberger und Schüller 2016), dass sie bei der Pflanzung nur wenig Feinwurzeln aufweisen. Dies macht sie speziell in trockenen Jahren anfällig für Ausfälle, weshalb eine Herbstpflanzung unbedingt zu empfehlen ist (Spornberger und Schüller 2016).

Funding Open access funding provided by University of Natural Resources and Life Sciences Vienna (BOKU).

Interessenkonflikt A. Spornberger, G. Osterc, E. Schüller und D. Noll geben an, dass kein Interessenkonflikt besteht.

Open Access Dieser Artikel wird unter der Creative Commons Namensnennung 4.0 International Lizenz veröffentlicht, welche die Nutzung, Vervielfältigung, Bearbeitung, Verbreitung und Wiedergabe in jeglichem Medium und Format erlaubt, sofern Sie den/die ursprünglichen Autor(en) und die Quelle ordnungsgemäß nennen, einen Link zur Creative Commons Lizenz beifügen und angeben, ob Änderungen vorgenommen wurden.

Die in diesem Artikel enthaltenen Bilder und sonstiges Drittmaterial unterliegen ebenfalls der genannten Creative Commons Lizenz, sofern sich aus der Abbildungslegende nichts anderes ergibt. Sofern das betreffende Material nicht unter der genannten Creative Commons Lizenz steht und die betreffende Handlung nicht nach gesetzlichen Vorschriften erlaubt ist, ist für die oben aufgeführten Weiterverwendungen des Materials die Einwilligung des jeweiligen Rechteinhabers einzuholen.

Weitere Details zur Lizenz entnehmen Sie bitte der Lizenzinformation auf http://creativecommons.org/licenses/by/4.0/deed.de.

\section{Literatur}

AMA (2018) Daten der biologisch bewirtschafteten Flächen in Österreich. Agrarmarkt Austria, Wien

Carrera M, Gomez-Aparisi J (2000) Pear rootstocks trials. Proceedings of the ISHS-th International Pear Symposium, Ferrara-Bologna, 04.-09.09.2000, S 100

Khazaei J, Chegini G, Bakhshiani M (2008) A novel alternative method for modelling the effect of air dry temperature and slice thickness on quality and drying kinetics of tomato slices: superposition technique. Dry Technol 26:759 
Mbabu P, Spethmann W (2005) Effect of length of cuttings, substrate $\mathrm{pH}$ and mineral nutrition on rooting of Pyrus communis cultivars. Eur J Hortic Sci 70:189-194

OECD (2005) Leitfaden zu objektiven Testmethoden zur Bestimmung der Qualität von Obst und Gemüse sowie Trocken- und getrockneten Erzeugnissen

Rueß F (2007) Birnensorten für den ökologischen Anbau. Öko-Obstbau 1:20-23

Spethmann W (2007) Increase of rooting success and further shoot growth by long cuttings of woody plants. Propag Ornam Plants 7:160-166

Spornberger A, Schüller E (2016) Influence of rootstocks compared to own-rooted pear trees on vegetative and generative characteristics under organic production conditions in eastern Austria. Erwerbsobstbau 58(1):1-9

Stanica F, Dumitrascu M, Peticila A (2000) Behavior of three pear varieties propagated "in vitro" and self-rooted on Tatura Trellis Canopy. Proceedings of the ISHS-8th International Pear Symposium, Ferrara-Bologna, 4-9.9.2000, S 202-203

Steinbauer L (2018) Das Match der Birnenunterlagen Quitten gegen Sämlinge endet 2:0. Teil 1 - Ertrags- und Wuchsverhalten. In: Haidegger Perspektiven, S 7-9
Thibault B, Hermann L (1982) Comportement agronomique de poiriers "Williams" cultivés sur leurs propres racines. Rev Hortic 230:57-60

Thybo K, Edelenbos M, Christensen L, Sørensen J, Thorup-Kristensen K (2006) Effect of organic growing systems on sensory quality and chemical composition of tomatoes. LWT Food Sci Technol 39:835

Tibiletti E (2001) Pero, dall'impianto alla raccolta. AZ Bio 2:44-48

Tomaz ZFP, Rodrigues AC, Verissimo V, Marafon AC, Herter FG, de Rufato AR (2009) Compatibility of pear cultivars on quinces rootstocks. Rev Bras Frutic 31:1211-1217

Weber H-J (2004) Birnenunterlagen - keine M9 in Sicht. DLR Rheinpfalz (KOGA), Bad Neuenahr-Ahrweiler

Wurm L, Harmer A, Darnhofer P, Lippitz M (2005) Leistungsprüfung verschiedener Apfelsorten bei biologischer und integrierter Produktion unter Berücksichtigung unterschiedlicher Baumstreifenpflege, Teil 3: Innere Fruchtqualität und Lagerverhalten. Mitt Klosterneuburg 55:162-176

Wurm L, Spornberger A, Gantar E-M, Kieler M (2014) Vergleich von selbstbewurzelten mit auf gängige Unterlagen veredelten Birnbäumen. Mitt Klosterneuburg 64:137-147 